\title{
A COMPACTNESS CONDITION \\ FOR SOLUTIONS OF ORDINARY DIFFERENTIAL EQUATIONS ${ }^{1}$
}

\author{
L. K. JACKSON
}

\begin{abstract}
It is proven that a sequence $\left\{y_{k}(x)\right\}$ of solutions of $y^{(n)}$ $=f\left(x, y, y^{\prime}, \ldots, y^{(n-1)}\right)$ with $\left\{y_{k}(x)\right\}$ uniformly bounded on a compact interval $[c, d]$ has a bounded total variation sequence $\left\{V_{c}^{d}\left(y_{k}\right)\right\}$ provided solutions of the differential equation extend and $n$-point boundary value problems have at most one solution.
\end{abstract}

It is a well-known property of linear differential equations that the uniqueness of the solution of a linear boundary value problem implies the existence of a solution of the problem. In the past several years there has been considerable interest in the question of to what extent similar results might hold for nonlinear differential equations, see for example, [1]-[7]. The following theorem, which was proven independently by Hartman [4] and Klaasen [7], is an example of one of the more important results that have been obtained.

THEOREM 1. Assume that for the differential equation

$$
y^{(n)}=f\left(x, y, y^{\prime}, \ldots, y^{(n-1)}\right), \quad n \geqslant 2,
$$

the following conditions are satisfied:

(A) $f\left(x, y, y^{\prime}, \ldots, y^{(n-1)}\right)$ is continuous on $(a, b) \times R^{n}$;

(B) all solutions of (1) extend to $(a, b)$;

(C) for any $a<x_{1}<x_{2}<\cdots<x_{n}<b$ and any solutions $y(x)$ and $z(x)$ of (1), $y\left(x_{i}\right)=z\left(x_{i}\right)$ for $1 \leqslant i \leqslant n$ implies that $y(x) \equiv z(x)$ on $\left[x_{1}, x_{n}\right]$; and

(D) if $[c, d]$ is a compact subinterval of $(a, b)$ and $\left\{y_{k}(x)\right\}$ is a sequence of solutions of (1) which is uniformly bounded on $[c, d]$, then there is a subsequence $\left\{y_{k_{i}}(x)\right\}$ such that $\left\{y_{k_{j}}^{(i)}(x)\right\}$ converges uniformly on $[c, d]$ for $0 \leqslant i \leqslant n-1$.

Then given any $a<x_{1}<x_{2}<\cdots<x_{n}<b$ and given any real numbers $c_{i}, 1 \leqslant i \leqslant n$, there is a solution $y(x)$ of $(1)$ with $y\left(x_{i}\right)=c_{i}$ for $1 \leqslant i \leqslant n$.

In [4] and [7] Theorem 1 is proven with the additional hypothesis that solutions of initial value problems are unique. Subsequently, in work that has

Received by the editors June 18, 1975.

AMS (MOS) subject classifications (1970). Primary 34B15; Secondary 34C10.

Key words and phrases. Boundary value problem, total variation, Kamke Convergence Theorem, Green's function, Schauder-Tychonoff Theorem.

1 This work was partially supported by NSF Grant MPS75-06174.

- American Mathematical Society 1976 
not yet been published, Klaasen has used the results in [8] to prove Theorem 1 as stated.

An interesting unsettled question with regard to Theorem 1 is the question of whether or not the compactness hypothesis (D) is implied by the other hypotheses. If $n=2$, it is easy to see that (A) and (B) alone imply (D). This is not the case for $n=3$. In fact the equation $y^{\prime \prime \prime}=-\left[y^{\prime}\right]^{3}$ satisfies $(\mathrm{A})$ and (B) on $(-\infty,+\infty) \times R^{3}$ for $n=3$ but the sequence $\left\{y_{k}(x)\right\}$ of solutions of the initial value problems

$$
y^{\prime \prime \prime}=-\left[y^{\prime}\right]^{3}, \quad y(0)=y^{\prime}(0)=0 \quad y^{\prime \prime}(0)=k
$$

for $k=1,2, \ldots$, is uniformly bounded on $(-\infty,+\infty)$ and does not contain a subsequence satisfying (D) on any compact interval. However, it is proven in [2] that for $n=3$ hypotheses (A), (B), and (C) do imply (D). It remains an open question as to whether or not this is the case for equation (1) of degree $n \geqslant 4$.

In the following theorem we prove that for equation (1) of arbitrary degree $n$ the hypotheses (A), (B), and (C) of Theorem 1 do imply a much weaker type of compactness condition for solutions of equation (1).

THEOREM 2. Assume that the differential equation (1) satisfies hypotheses (A), (B), and (C) of Theorem 1. Then, if $[c, d]$ a compact subinterval of $(a, b)$ and $\left\{y_{k}(x)\right\}$ is a sequence of solutions of (1) which is uniformly bounded on $[c, d]$, it follows that the sequence $\left\{V_{c}^{d}\left(y_{k}\right)\right\}$ of total variations of the functions $y_{k}(x)$ on $[c, d]$ is bounded.

Proof. Assume on the contrary that (1) satisfies (A), (B), and (C) but that there is a compact interval $[c, d] \subset(a, b)$ and a sequence $\left\{y_{k}(x)\right\}$ of solutions of (1) with $\left|y_{k}(x)\right| \leqslant M$ on $[c, d]$ for all $k \geqslant 1$ and with $\left\{V_{c}^{d}\left(y_{k}\right)\right\}$ unbounded. Then by choosing a subsequence and relabeling, if necessary, we can assume $V_{c}^{d}\left(y_{k}\right) \rightarrow+\infty$ as $k \rightarrow \infty$.

The condition $V_{c}^{d}\left(y_{k}\right) \rightarrow+\infty$ implies that $\lim _{k \rightarrow+\infty} \sum_{i=0}^{n-1}\left|y_{k}^{(i)}(x)\right|=+\infty$ uniformly on $[c, d]$. For, if it were not the case that $\lim _{k \rightarrow \infty} \sum_{i=0}^{n-1}\left|y_{k}^{(i)}(x)\right|$ $=+\infty$ uniformly on $[c, d]$, then by the Kamke Convergence Theorem [9, p. 14] a subsequence $\left\{y_{k_{j}}(x)\right\}$ could be chosen such that $\left\{y_{k_{j}}^{(i)}(x)\right\}$ would converge uniformly on $[c, d]$ for each $i=0,1, \ldots, n-1$. Obviously, this would contradict $V_{c}^{d}\left(y_{k}\right) \rightarrow+\infty$.

Now consider the boundary value problem

$$
y^{(n)}=f\left(x, y, y^{\prime}, \ldots, y^{(n-1)}\right), \quad y\left(x_{i}\right)=\alpha, \quad 1 \leqslant i \leqslant n,
$$

where $a<x_{1}<x_{2}<\cdots<x_{n}<b$ and $\alpha$ is a constant. Let $G(x, s)$ be the Green's function for the boundary value problem

$$
y^{(n)}=0, \quad y\left(x_{i}\right)=0, \quad 1 \leqslant i \leqslant n .
$$

Then it follows from hypothesis (A) alone that $y(x) \in C^{(n)}\left[x_{1}, x_{n}\right]$ and is a solution of boundary value problem (2) if and only if $y(x) \in C^{(n-1)}\left[x_{1}, x_{n}\right]$ and is a solution of the integral equation 


$$
y(x)=\alpha+\int_{x_{1}}^{x_{n}} G(x, s) f\left(s, y(s), y^{\prime}(s), \ldots, y^{(n-1)}(s)\right) d s .
$$

From an application of the Schauder - Tychonoff Fixed Point Theorem, we conclude that there is a fixed $\delta>0$ such that for any $\alpha$ with $|\alpha| \leqslant M$ and any points $c \leqslant x_{1}<x_{2}<\cdots<x_{n} \leqslant d$ with $x_{n}-x_{1} \leqslant \delta$, integral equation (3), hence boundary value problem (2), has a solution $y(x)$ with $|y(x)| \leqslant M+1$ on $\left[x_{1}, x_{n}\right]$ and $\left|y^{(i)}(x)\right| \leqslant 1$ on $\left[x_{1}, x_{n}\right]$ for $1 \leqslant i \leqslant n-1$. Here $M$ is the bound on the sequence $\left\{y_{k}(x)\right\}$ on $[c, d]$.

Let $k_{0}$ be such that $\sum_{i=0}^{n-1}\left|y_{k}^{(i)}(x)\right|>M+n$ on $[c, d]$ for $k \geqslant k_{0}$. Then for $k \geqslant k_{0}$ the graph of $y=y_{k}(x)$ can intersect a line $y=\alpha,|\alpha| \leqslant M$, in at most $n-1$ distinct points in a subinterval of $[c, d]$ of length not exceeding $\delta$ where $\delta>0$ is as above. For, if $y_{k}\left(x_{i}\right)=\alpha$ at points $x_{i}, 1 \leqslant i \leqslant n$, with $c \leqslant x_{1}$ $<x_{2}<\cdots<x_{n} \leqslant d$ and $x_{n}-x_{1} \leqslant \delta$, it would follow from the uniqueness hypothesis $(\mathrm{C})$ that $y_{k}(x)$ would coincide on $\left[x_{1}, x_{n}\right]$ with the solution obtained using the fixed point theorem, which would contradict $\sum_{i=0}^{n-1}\left|y_{k}^{(i)}(x)\right|>M$ $+n$ on $\left[x_{1}, x_{n}\right]$. Thus, if $m$ is the integer such that $(m-1) \delta \leqslant d-c<m \delta$, then for $k \geqslant k_{0}$ the graph of $y=y_{k}(x)$ cannot intersect a line $y=\alpha,|\alpha|$ $\leqslant M$, in more than $(n-1) m$ distinct points. For $-M \leqslant \alpha \leqslant+M$, let $h_{k}(\alpha)$ be the number of distinct points $x \in[c, d]$ such that $y_{k}(x)=\alpha$. Then it follows [10, p. 270] that $V_{c}^{d}\left(y_{k}\right)=\int_{-M}^{+M} h_{k}(\alpha) d \alpha$. This leads to the contradiction that $V_{c}^{d}\left(y_{k}\right) \leqslant 2 M(n-1) m$ for all $k \geqslant k_{0}$ and the proof of the theorem is complete.

COROLlARY. Assume that equation (1) satisfies hypotheses (A), (B), and (C) of Theorem 1. Then, if $[c, d]$ is a compact subinterval of $(a, b)$ and if $\left\{y_{k}(x)\right\}$ is a sequence of solutions of (1) which is uniformly bounded on $[c, d]$, there is a subsequence $\left\{y_{k_{j}}(x)\right\}$ which converges pointwise on $[c, d]$ and $z(x)$ $\equiv \lim _{k_{j} \rightarrow \infty} y_{k_{j}}(x)$ is of bounded variation on $[c, d]$.

Proof. The result follows from the Helly Theorem [11, p. 398] and the fact that $\left\{y_{k}(x)\right\}$ and $\left\{V_{c}^{d}\left(y_{k}\right)\right\}$ are bounded.

Schrader [12] has proven that, if $\left\{y_{k}(x)\right\}$ is a uniformly bounded sequence of functions on a compact interval $[c, d]$ and if the functions $y_{k}(x)$ satisfy only the uniqueness hypothesis $(C)$ on $[c, d]$, then there is a subsequence which converges pointwise on $[c, d]$.

\section{REFERENCES}

1. A. Lasota and Z. Opial, On the existence and uniqueness of solutions of a boundary value problem for an ordinary second-order equation, Colloq. Math. 18 (1967), 1-5. MR 36 \#2871.

2. L. K. Jackson and K. Schrader, Existence and uniqueness of solutions of boundary value problems for third order differential equations, J. Differential Equations 9 (1971), 46-54. MR 42 \#4813.

3. K. Schrader and P. Waltman, An existence theorem for nonlinear boundary value problems, Proc. Amer. Math. Soc. 21 (1969), 653-656. MR 39 \#533.

4. P. Hartman, On N-parameter families and interpolation problems for nonlinear ordinary differential equations, Trans. Amer. Math. Soc. 154 (1971), 201-226. MR 46 \#35.

5. P. Waltman, Existence and uniqueness of solutions of boundary value problems for two dimensional systems of nonlinear differential equations, Trans. Amer. Math. Soc. 153 (1971), 223-239. MR 42 \#3347. 
6. L. Shampine, Existence and uniqueness for nonlinear boundary value problems, J. Differential Equations 5 (1969), 346-351. MR 39 \# 534.

7. G. Klaasen, Existence theorems for boundary value problems for nth order ordinary differential equations, Rocky Mountain J. Math. 3 (1973), 457-472.

8. Continuous dependence for n-point boundary value problems, SIAM J. Appl. Math. 19 (1975), 99-102.

9. P. Hartman, Ordinary differential equations, Wiley, New York, 1964. MR 30 \# 1270.

10. E. Hewitt and K. Stromberg, Real and abstract analysis. A modern treatment of the theory of functions of a real variable, Springer-Verlag, New York, 1965. MR 32 \# 5826.

11. A. E. Taylor, General theory of functions and integration, Blaisdell, Waltham, Mass., 1965. MR 31 \#2358.

12. K. Schrader, A generalization of the Helly selection theorem, Bull. Amer. Math. Soc. 78 (1972), 415-419. MR 45 \#8788.

Department of Mathematics, University of Nebraska, Lincoln, Nebraska 68588 